\title{
EFECTO DE UN PROGRAMA DE EJERCICIO AERÓBICO Y UN PROGRAMA DE CIRCUITO CON PESAS SOBRE LA CALIDAD DE VIDA, DISNEA Y RESISTENCIA CARDIORRESPIRATORIA EN SUJETOS CON ENFERMEDAD PULMONAR CRÓNICA
}

\author{
Delgado Acosta Henry ${ }^{1}$ y Acuña Corrales Ernesto ${ }^{2}$, \\ ${ }^{1}$ Hospital Calderón Guardia, henryada76@ hotmail.com \\ ${ }^{2}$ EKA Centro de Salud Física, ernesvito23@gmail.com \\ 2007
}

RESUMEN

\begin{abstract}
El objetivo de este estudio fue determinar el efecto de un programa de ejercicio aeróbico y un programa de circuito con pesas sobre la calidad de vida, disnea y resistencia cardiorrespiratoria en sujetos con Enfermedad Pulmonar Crónica (EPC). Metodología: el programa de rehabilitación se hizo durante ocho semanas, se realizó un pretest y un postest, se cumplió con una sesión semanal presencial, y las otras dos domiciliarias, se tuvo comunicación directa vía telefónica los días que realizaban el ejercicio en la casa. Instrumentos: el cuestionario St. George Respiratory Questonnaire para medir calidad de vida, la escala de Borg para medir la disnea y el test de caminata de los 6 min. para medir la resistencia cardiorrespiratoria. Sujetos: participaron 38 personas con diagnóstico previo de EPC, 18 hombres y 20 mujeres; 27 sujetos con patología obstructiva y 11 sujetos con restrictiva., con una edad promedio de $69.8 \pm 9.34$ años, divididos aleatoriamente en dos grupos, uno que realizó ejercicio aeróbico (22 sujetos) y otro que ejecutó ejercicio aeróbico y de contrarresistencia con pesas (16 sujetos). Análisis estadístico: ANOVA mixta de cuatro vías $(2 \times 2 \times 2 \times 2)$ para las variables mediciones, sexo, tratamiento y patología. Resultados: se encontraron diferencias significativas entre mediciones para la variable disnea (pre: $7.18 \pm 0.69$ puntos y post: $4.89 \pm 0.68$ puntos) $(F=228.770 ; p<$ $0,05)$, calidad de vida (pre: $59.68 \pm 7.50$ puntos y post $81.75 \pm 7.33$ puntos) $(F=228.770 ; p<0,05)$, resistencia cardiorrespiratoria (pre 384,95 $\pm 51,02$ mts. y post $432 \pm 37,41 \mathrm{mts}$.) $(F: 59.9 ; p<0.01)$, pero no se encontraron diferencias por sexo, actividad ni enfermedad ( $p>0,05)$, ni interacción entre las variable ( $p>0,05)$. Conclusión: la realización de ejercicio físico aeróbico y anaeróbico mejoran el grado de disnea, la calidad de vida y resistencia cardiorrespiratoria en pacientes con EPC.
\end{abstract}

PALABRAS CLAVES: enfermedad pulmonar crónica, ejercicio aeróbico, ejercicio anaeróbico, pesas, capacidad cardiorrespiratoria, disnea, calidad de vida.

\section{EFFECT OF AN AEROBIC EXERCISE PROGRAM AND A WEIGHT CIRCUIT PROGRAM ON THE QUALITY OF LIFE, DYSPNEA, AND CARDIORESPIRATORY RESISTANCE IN SUBJECTS WITH CHRONIC PULMONARY DISEASE}

\section{ABSTRACT}

The objective of this study was to determinate the effect of an aerobic exercise program and a weight circuit program on the quality of life, dyspnea and cardiorespiratory resistance in subjects with Chronic Pulmonary Disease (CPD). Methodology: the rehabilitation program was conducted for eight weeks, including a pre-test and a post-test, one on-site supervised session per week and two home sessions with direct telephone communication. Instruments: the St. George Respiratory Questionnaire was used to measure quality of life, the Borg Scale to measure dyspnea, and the Six Minute Walk Test to measure cardiorespiratory resistance. Subjects: 38 patients previously diagnosed with CPD, 18 men and 20 women; 27 subjects with an obstructive pathology and 11 with a restrictive pathology, with an average age of $69.8 \pm 9.34$ years. Subjects were randomly divided into two groups: one did aerobic exercises (22 subjects) and the other one did aerobic exercises and resistance training (16 subjects). Statistical Analysis: Four-Way ANOVA (2x2x2x2) for the variables sex, treatment, and pathology. Results: significant differences were found between measurements of the following variables: dyspnea (pre-test: $7.18 \pm 0.69$ points and post-test: $4.89 \pm 0.68$ points) $(F=228.770 ; p<$ $0.05)$, quality of life (pre-test: $59.68 \pm 7.50$ points and post-test $81.75 \pm 7.33$ points $)(F=228.770 ; p<0.05)$, cardiorespiratory resistance (pre-test $384.95 \pm 51.02 \mathrm{mts}$. and post-test $432 \pm 37.41 \mathrm{mts}$.) $(F: 59.9 ; p<0.01)$. However, no differences were found in $s e x$, activity program or disease $(p>0.05)$, or any interaction between variables $(p>0.05)$. Conclusion: aerobic and anaerobic exercises improve the degree of dyspnea, the quality of life, and the cardiorespiratory resistance in CPD patients.

KEY WORDS: chronic pulmonary disease, aerobic exercises, anaerobic exercises, weight lifting, cardiorespiratory capacity, dyspnea, quality of life. 


\section{INTRODUCCIÓN}

Las enfermedades pulmonares crónicas están entre las mayores causas de morbi-mortalidad. Las estadísticas de salud sobreestiman estas patologías porque la dificultad para definirlas y diagnosticarlas es muy compleja (Pallares, 2003).

El desarrollo del compromiso clínico, funcional y del círculo vicioso, que se deriva de la disnea, la fatigabilidad, la limitación crónica, severa del flujo aéreo y la disminución incapacitante de la capacidad vital en el paciente con enfermedad restrictiva, independientemente de su etiología, dificultan el trabajo del neumópata y los conduce al desentrenamiento y a la incapacidad física, con la consecuente repercusión en la calidad de vida. Con el propósito de evitar o romper este círculo vicioso, diferentes consensos y guías clínicas recomiendan la rehabilitación pulmonar (RP) a partir de etapas moderadas de la enfermedad (Hernández, Rivas, Núñez y Álvarez, 2000 y Ries, 2004).

El objetivo principal de un programa de RP es restaurar al máximo en el paciente su independencia y funcionamiento en la comunidad. Esto implica mayor responsabilidad de ellos, porque deben tener mayor conocimiento de su enfermedad y responsabilizarse del cuido de su salud (Hernández y otros, 2000 y Ries, 2004). Estudios previos han demostrado la importancia de la rehabilitación pulmonar, debido a que se han encontrado incrementos en la tolerancia al ejercicio, mejora en la calidad de vida, la frecuencia cardiaca y la presión arterial, disminución en la sintomatología y en las consultas médicas. La mayoría de estos estudios son con grupos pequeños de pacientes, observacionales y con grupos que no han sido aleatorizados y por lo general utilizando predominantemente el ejercicio aeróbico, sin embargo, pocos estudios se han referido al entrenamiento de contrarresistencia (Weg, 1985; Phillips, Benton, Wagner y Riley, 2006; Ries, 2004; Williams y Maresh, 2005). Además, los programas de ejercicio físico contribuyen a mejorar las funciones del sistema cardiovascular, músculo esquelético y pulmonar. No obstante, las acciones sobre el organismo son más amplias, bien por el efecto propio del ejercicio o a través de las modificaciones de los factores de riesgo cardiovasculares inducidas por el entrenamiento (Prat, 2003). Por ejemplo, Weg (1985) realizó un trabajo de ejercicio terapéutico en pacientes con EPOC severo donde sin incluir el trabajo de contrarresistencia específico, se mejoró la tolerancia al ejercicio, así como las habilidades mecánicas en los músculos que fueron entrenados. Asimismo, Casaburi y otros (1997), realizaron un estudio con ejercicio aeróbico a alta intensidad y encontraron mejorías significativas en la resistencia cardiorrespiratoria, capacidad vital forzada en reposo, consumo máximo de oxígeno y tolerancia al ejercicio.

Grassino (1989), recomienda ambos tipos de entrenamiento, pero que tanto el trabajo aeróbico como el de contrarresistencia deberían inducir al aumento de la fuerza de músculos espiratorios para producir una tos eficaz y, de músculos inspiratorios para aumentar su reserva de fuerza, esto da lugar a una mejor calidad de la vida y una disminución en la hospitalización y de los costos.

En cuanto al ejercicio contrarresistencia, uno de estos estudios fue el realizado por Rooyackers y Folgering (1998), quienes utilizaron entrenamiento de fuerza isométrico y dinámico en conjunto con actividades de la vida diaria, cinco días a la semana durante diez semanas, y encontraron mejorías significativas en la capacidad máxima de trabajo $(p<0,05)$. Otro estudio realizado por Phillips y otros (2006), utilizó en un grupo RP y en otro grupo RP se realizo más entrenamiento de fuerza y encontraron que las personas que trabajaron solo RP disminuyeron la fuerza tanto en tren inferior como en tren superior y el grupo que realizó RP y entrenamiento de fuerza mejoraron significativamente $(\mathrm{p}<0,05)$, además en cuanto a la capacidad funcional este último tuvo mejorías significativas en cinco de siete test aplicados $(\mathrm{p}<0.05)$.

Por lo anterior expuesto y debido a las escasas posibilidades de efectuar un programa adecuado de rehabilitación pulmonar, en los últimos estudios, Hernández y otros, 2000 y Ries (2004), han 
considerado que la educación del usuario esta cubriendo algunos aspectos básicos respecto a su enfermedad, a la importancia de su adherencia al tratamiento y a la consulta precoz durante las exacerbaciones, asociado a ejercicio físico, idealmente supervisado, con la carga de entrenamiento que el paciente sea capaz de manejar, permite obtener beneficios clínicos importantes desde el punto de vista del individuo, razones por las cuales justifica el propósito de este estudio que es determinar el efecto de un programa de ejercicio aeróbico y un programa de circuito con pesas sobre la calidad de vida, disnea y capacidad cardiorrespiratoria en sujetos con EPC.

\section{METODOLOGÍA}

Sujetos: En el estudio participaron 38 sujetos, con una edad promedio de $69.8 \pm 9.34$ años, divididos aleatoriamente en dos grupos, uno que realizó ejercicio aeróbico (GEA) con 22 sujetos y otro que hizo ejercicio aeróbico combinado con ejercicio de contrarresistencia con pesas (GEAP) con 16 sujetos. En general 18 eran hombres y 20 eran mujeres. Todos los participantes eran referidos a la consulta de rehabilitación con diagnóstico previo de EPC y tenían que cumplir con los siguientes criterios de inclusión: personas con limitación respiratoria crónica quienes a pesar de manejo médico óptimo, están disneicos, tienen reducida tolerancia al ejercicio o experimentan restricción en actividades. Indicaciones comunes, ansiedad al hacer ejercicio, disnea con las actividades; limitación con actividades sociales, de recreación, obligaciones dentro o fuera de casa, actividades de la vida diaria (básicas, complementarias), pérdida de independencia. Los diagnósticos incluidos fueron: enfermedad pulmonar crónica, asma, enfermedades de la pared torácica, fibrosis quística, enfermedad pulmonar intersticial, fibrosis post síndrome de distress respiratorio del adulto (ARDS) y cáncer pulmonar. Para efectos del estudio fueron analizados también en dos grupos, esto de acuerdo al criterio de patología obstructiva (27 sujetos) y restrictiva (11 sujetos).

Instrumentos: se determinó la calidad de vida por medio del cuestionario St. George Respiratory Questonnaire, compuesto por 50 ítems, el cual reporta un coeficiente alfa de Cronbach de 0.94 para la variable calidad de vida general y según las subescalas 0.72 para síntomas, 0.89 para actividad y 0.89 para impacto; para efectos de este estudio solo se hizo análisis general, no por subescalas (Ferrer y otros, 1996 y Aguilar, 2000). El cuestionario Saint George (CRSG) fue creado con el fin evaluarlo en usuarios con enfermedad pulmonar obstructiva crónica y asma (Aguilar, 2000), el cual ha sido de de extrema utilidad en la valoración de actividad e impacto de la enfermedad en la vida cotidiana.

Posteriormente se utilizó la escala modificada de Borg (con una confiabilidad de $r=0.99$ ) (Sansores, 2006), para medir el grado de disnea, con una escala de 1 a 10, donde se ubica el grado que presenta posterior a la caminata de 6 minutos (con una confiabilidad de $\mathrm{r}=0.99, \mathrm{p}=<$ 0.01) (Guyatt, 1995). . También se utilizó la prueba de caminata de los 6 minutos para medir la resistencia cardiorrespiratoria, la cual se ha aplicado desde 1968 como un excelente predictor de la morbimortalidad en sujetos neumópatas, así como para evaluar resultados de tratamientos de rehabilitación pulmonar en sujetos con EPOC y otras neuropatías, la cual se realiza en terreno plano, en un tramo de 30 metros, marcado por conos cada 5 metros (Luna, 2000).

Además se realizó medición de signos vitales y oximetría de pulso para controlar la saturación arterial de oxígeno, como medio preventivo y evitar complicaciones potenciales de tipo cardiorrespiratorias.

Procedimiento: el programa de rehabilitación se hizo inicialmente como lo recomienda Clark, Cochrane y Mackay (1996), durante 8 semanas, con una medición antes de iniciarlo y otra al finalizar este.

La división del grupo se hizo de forma aleatoria, ya que las personas que iban a consulta médica los días pares se les prescribió el ejercicio aeróbico con el circuito de pesas y los sujetos que 
asistían a consulta médica los días impares, solo se les prescribió ejercicio aeróbico. La metodología de trabajo tanto para el grupo GEA como para el grupo GEAP, fue la siguiente: se realizó una sesión semanal presencial, y las otras dos domiciliarias, se estableció comunicación directa vía telefónica los días que realizaban el ejercicio en la casa.

En cuanto a las sesiones de ejercicio aeróbico, las intensidades que se trabajaron oscilaron entre un 40 a $60 \%$ del $\mathrm{VO}_{2 \text { máx. }}$ Y se monitoreó por medio de la escala de percepción de esfuerzo de Borg, por otro lado, el trabajo de contrarresistencia realizado por los sujetos se compuso de ejercicios para tren superior (bíceps y elevaciones laterales) y para tren inferior (extensión de piernas). La cantidad de peso y de ejercicios se modificaba de acuerdo a la tolerancia al ejercicio del paciente y para eso se utilizó la escala de percepción de esfuerzo de Borg. Asimismo, para las sesiones domiciliarias las pesas fueron elaboradas por los mismos sujetos con la supervisión de especialistas.

Análisis estadístico: se aplicó estadística descriptiva y ANOVA mixta de cuatro vías $(2 \times 2 \times 2 \times 2)$ para las mediciones, tipo de tratamiento (GEA y GEAP), sexo y patología. Los datos fueron procesados con el programa estadístico SPSS, versión 8.0 para Windows.

\section{RESULTADOS}

A continuación se describen los resultados obtenidos en el presente estudio: Gráfico 1. Cambios en la variable disnea en dos tipos de ejercicio
Aeróbico y anaeróbico en sujetos con EPOC

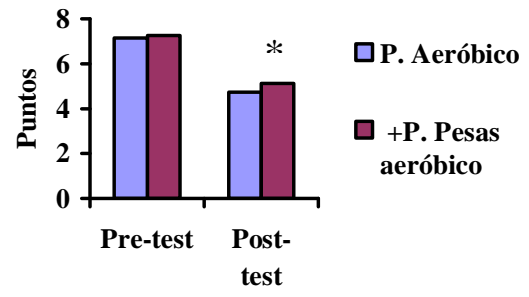

*F: 228.77; $\mathrm{p}<0.01$

De acuerdo al gráfico 1, y por medio de la prueba ANOVA mixta de cuatro vías (2x2x2x2) para la variable disnea, se puede observar que se encontraron diferencias estadísticamente significativas entre mediciones, pretest $(7.18 \pm 0.7$ puntos $)$ y post $(4.89 \pm 0.69$ puntos $)(\mathrm{F}=$ 228.770; $\mathrm{p}<0,05)$; sin embargo no se encontraron diferencias estadísticas significativas por sexo $(F=3,584 ; p>0,05)$, actividad $(F=1,670 ; p>0,05)$, enfermedad $(F=1,374 ; p>0.05)$, ni interacción entre: actividad $*$ sexo $(\mathrm{F}=1,106 ; \mathrm{p}>0,05)$, actividad $*$ enfermedad $(\mathrm{F}=0,023$; $\mathrm{p}>0,05)$, sexo $*$ enfermedad, $(\mathrm{F}=2,168 ; \mathrm{p}>0,05)$, actividad $*$ sexo $*$ enfermedad $(\mathrm{F}=0,153$; $\mathrm{p}>0,05)$. 


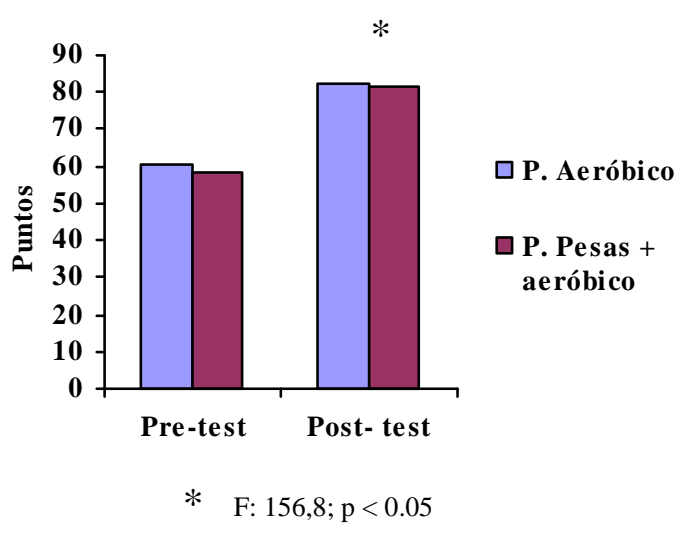

De acuerdo al gráfico 2, y por medio de la prueba ANOVA mixta de cuatro vías $(2 \times 2 \times 2 \times 2)$ para la variable Calidad de Vida, se puede observar que se presentaron diferencias estadísticamente significativas para los efectos de medición pre $(59.68 \pm 7.50$ puntos $)$ y post $(81.75 \pm 7.33)(\mathrm{F}=$ 228.770; $\mathrm{p}<0,05)$; sin embargo no se encontraron diferencias estadísticas significativas entre sexo $(F=0.002 ; p>0,05)$, actividad $(F=0.551 ; p>0,05)$, enfermedad $(F=2.704 ; p>0.05)$, ni interacción entre: actividad $*$ sexo $(\mathrm{F}=0.148 ; \mathrm{p}>0,05)$, actividad $*$ enfermedad $(\mathrm{F}=1.041$; $\mathrm{p}>0,05)$, sexo $*$ enfermedad, $(\mathrm{F}=0.096 ; \mathrm{p}>0,05)$, actividad $*$ sexo $*$ enfermedad $(\mathrm{F}=0.742$; $\mathrm{p}>0,05)$.

Gráfico 1. Cambios en la variable capacidad cardiorrespiratoria en dos tipos de ejercicio aeróbico y anaeróbico en sujetos con EPOC

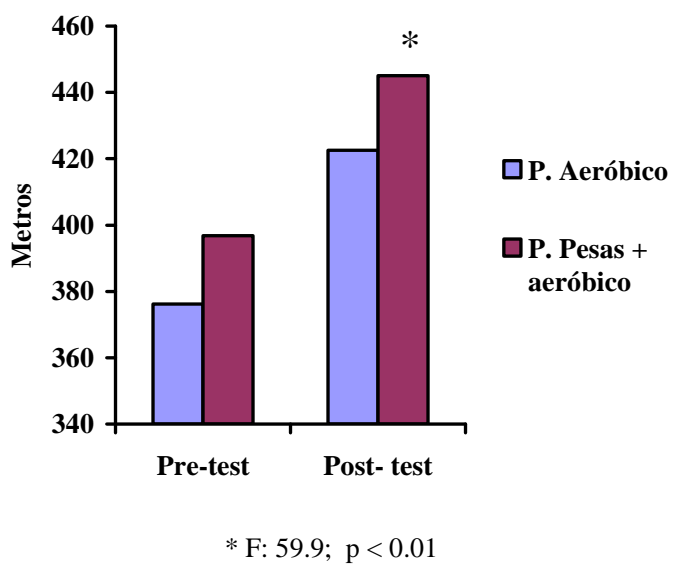

De acuerdo al gráfico 1 , y por medio de la prueba ANOVA mixta de cuatro vías $(2 \times 2 \times 2 \times 2)$ para la variable resistencia cardiorrespiratoria, se puede observar que se encontraron diferencias estadísticamente significativas para los efectos de medición pre $(384,95 \pm 51,02 \mathrm{mts})$ y post $(432 \pm 37,41 \mathrm{mts})(\mathrm{F}: 59.9 ; \mathrm{p}<0.01)$; sin embargo no se encontraron diferencias estadísticas significativas por sexo $(F=0,044 ; p>0,05)$, actividad $(F=1,102 ; p>0,05)$, enfermedad $(F=$ $0,741 ; \mathrm{p}>0.05)$, ni interacción entre: actividad $*$ sexo $(\mathrm{F}=0,460 ; \mathrm{p}>0,05)$, actividad $*$ enfermedad $(\mathrm{F}=3,289 ; \mathrm{p}>0,05)$, sexo * enfermedad, $(\mathrm{F}=0,130 ; \mathrm{p}>0,05)$, actividad * sexo * enfermedad $(\mathrm{F}=1,766 ; \mathrm{p}>0,05)$.

\section{DISCUSIÓN}

El objetivo principal de esta investigación fue determinar el efecto de un programa de ejercicio aeróbico y uno de circuito con pesas sobre la calidad de vida, disnea y capacidad cardiorrespiratoria en sujetos con Enfermedad Pulmonar Crónica.

De acuerdo con los resultados obtenidos en este estudio, se determinó que ambos tratamientos mejoraron las variables disnea, resistencia cardiorrespiratoria y calidad de vida entre 
mediciones. En cuanto a la variable disnea los resultados obtenidos en este estudio son respaldados por estudios previos como los de Lacasse y otros (2007) quienes encontraron una disminución significativa en la disnea, cuando individuos con EPC, fueron sometidos a un programa de rehabilitación pulmonar y física, de igual forma Padula y Yeaw (2006), quienes en una investigación demostraron que un protocolo estándar del $30 \%$ o más alto para una duración de 20 a 30 minutos por día durante 10 a 12 semanas mejora disnea y la fuerza inspiratoria, además de la resistencia inspiratoria, este hallazgo encontrado podría deberse a lo indicado por Grassino (1989), quien explica que tanto el trabajo aeróbico como el de contrarresistencia, inducen al aumento de la fuerza de músculos espiratorios para producir una tos eficaz y de músculos inspiratorios para aumentar su reserva de fuerza, lo que a su vez da lugar a una mejor calidad de la vida y una disminución en la disnea.

En cuanto a la variable capacidad cardiorrespiratoria que indican este estudio, muestra una mejora significativa, lo que es apoyado por otras investigaciones como la realizada por Hsieh y otros (2007) donde han demostrado un aumento en la resistencia cardiorrespiratoria sometiéndolos a trabajo de ejercicio aeróbico, asimismo, Casaburi y otros (1997), realizaron un estudio con ejercicio aeróbico a alta intensidad y encontraron mejorías significativas en la resistencia cardiorrespiratoria, consumo máximo de oxígeno y tolerancia al ejercicio, lo que se podría relacionarse con lo encontrado por Prat (2003), quien indica que los programas de ejercicio físico contribuyen a mejorar las funciones del sistema cardiovascular, músculo esquelético y pulmonar. Esto confirma que la ruptura del ciclo de inactividad en este tipo de sujetos con este padecimiento, es fundamental para la reintegración del individuo al medio (Celli, 2002a).

En cuanto a la variable de calidad de vida también se encontraron mejorías significativas, al igual que lo indicado por Celli (2002b), quien muestra que estos sujetos mejoran debido a que disminuyen la disnea, ya que se reduce el sedentarismo, aumenta la fuerza muscular, lo que ayuda a optimizar la alimentación al mejorar el patrón respiratorio, lo que lleva a reintegrarlos al máximo en un rol social, en el que se sientan útiles y son independientes en su vida cotidiana.

Al buscar las razones de por qué no hubo diferencias entre tratamientos en la variable capacidad cardiorrespiratorio, pudo deberse a que según el ACSM (2006) el aumento en el componente cardiorrespiratorio por la práctica de circuito es solamente de un $5 \%$ y esta es la única diferencia en el tratamiento de un grupo a otro. En relación a la variable disnea y calidad de vida, según Grassino (1989) ambos tratamientos son beneficiosos para las anteriores variables. Pues no se encontraron diferencias en cuanto a sexo. Las semanas que se trabajo con la población de investigación fueron oportunas para evidenciar que en este tipo de paciente se da una mejoría, la cual se evidencia en su desempeño diario pero, que es poco tiempo para hacer una diferenciación entre la respuesta de distintos sexos, dado que se amerita por lo menos un lapso de 6 meses para describir una diferenciación fidedigna, además menciona que en un lapso de 8 a 10 semanas lo que se hace es un reacondicionamiento general y las respuestas pueda que no sean las adecuadas en ese tiempo.

\section{CONCLUSIONES}

La realización de ejercicio físico aeróbico y anaeróbico mejoran el grado de disnea, la calidad de vida y resistencia cardiorrespiratoria en pacientes con enfermedad pulmonar crónica.

Que ambos programas son beneficiosos para los pacientes con EPOC.

Ambos tratamientos pueden ser beneficiosos para la inserción de estos pacientes en la vida social y además logran la independencia en sus actividades cotidianas. 


\section{REFERENCIAS}

ACSM (2006). Guidelines for Exercise Testing and Prescription. Sétima edición. Estados Unidos: Editorial Lippincott Williams \& Wilkins.

Aguilar, G. (2000). Reproducibilidad del Cuestionario Respiratorio Saint George en la Versión al Español, en pacientes Mexicanos con Enfermedad Pulmonar Obstructiva Crónica. Revista Instituto Nacional de Enfermedades Respiratorias México, 12 (13), 85-95.

Casaburi, R., Porszasz, J., Burns, M.R., Carithers, E.R., Chang, R.S. y Cooper, C.B. (1997). Physiologic benefits of exercise training in rehabilitation of patients with severe chronic obstructive pulmonary disease. Am. J. Respir. Crit. Care Med, 155 (5), 1541-1551.

Celli, BR. (2002a). American Thoracic Society. Standards for the diagnosis and care of patients with chronic obstructive pulmonary disease. American Journal of Respiratory Critical Care, 195, 77-120.

Celli, BR. (2002b). Clinical commentary. Pulmonary rehabilitation in patients with COPD. American Journal Respiratory Critical Care, Med, 861-864.

Clark, C.J., Cochrane, L. y Mackay, E. (1996). Low intensity peripheral muscle conditioning improves exercise tolerance and breathlessness in COPD. Eur Respir J, 9(12), 2590-6.

Ferrer, M., Alonso, J., Prieto, L., Plaza, V., Monsó, E., Marrades, R, Aguar, M.C., Khalaf, A. y Antó, J.M. (1996). Validity and reliability of the St George's Respiratory Questionnaire after adaptation to a language and culture: the Spanish example. Eur Respir J, 9, 11601166.

Grassino, A. (1989). Effects inspiratory muscle training in COPD patients. Eur Respir J Suppl, 7, 581s-586s.

Guyatt, G.H., Sullivan, M.J. y Thompson, P.J. (1995). The 6 minutes walk: a new measure of exercise capacity in patients with chronic heart failure. Can Med Assoc J, 132: 919-923.

Hernández, G., Rivas, E., Núñez, H. y Álvarez, G., (2000). Rehabilitación Cardiopulmonar. 4 Rev. Cubana de Cardiología, 14 (1), 48-54.

Hsieh, M.J., Lan, C.C., Chen, N.H-, Huang, C.C., Wu, Y.K., Cho, H.Y. y Tsai, Y.H. (2007). Effects of high-intensity exercise training in a pulmonary rehabilitation programme for patients with chronic obstructive pulmonary disease. Respirology, 12(3), 381-8.

Lacasse, Y., Brosseau, L., Milne, S., Martin, S., Wong, E., Guyatt, G.H. y Goldstein, R.S. (2007). Pulmonary rehabilitation for chronic obstructive pulmonary disease. Fuente: http//www.Mrw.interscience.wiley.com/Cochrane/clsysrew/articles/framehtml. Fecha de acceso: 12 de junio del 2007.

Luna, P. (2000). Estandarización de la prueba de caminata de 6 minutos. Revista Instituto Nacional de Enfermedades Respiratorias México, 14 (13), 205-210.

Padula, C. y Yeaw, E. Inspiratory muscle training: Integrative review. Research and Theory for Nursing Practice. 20(4):291-304. 2006.

Pallares, V. (2003). Ejercicios y Fisioterapia para el tratamiento del asma. España: Editorial Científico Técnica. 
Phillips, W.T., Benton, M.J., Wagner, C. L. y Riley, C. (2006). The effect of single set resistance training on strength and functional fitness in pulmonary rehabilitation patients. J Cardiopulm Rehabil, 26(5), 330-7.

Prat, T. (2003). Prescripción de actividad física en pacientes con cardiopatía coronaria. $\left(2^{\circ}\right.$ ed). Barcelona: Paidotribo, 163-88.

Ries, A.L. (2004). Position paper of the American Association of Cardiovascular and Pulmonary Rehabilitation. Scientific basis of pulmonary rehabilitation. Journal of Cardiopulmonary Rehabilitation. 10: 418-41.

Rooyackers, J.M. y Folgering, H.T. (1998). Cardio-respiratory load of exercise training in patients with severe COPD. Int J Rehabil Res, 21(3), 259-71.

Sansores, R. (2006). Mecanismos de la disnea. Fuente: http//www.medigrafic.com/pdfs/neumo/net.2006/. Fecha de acceso: 12 de mayo del 2007.

Smith, M. y Van Ball. (2000). Rehabilitación cardiovascular y respiratoria. España: Ediciones Harcourt.

Weg, J.G. (1985). Therapeutic exercise in patients with chronic obstructive pulmonary disease. Cardiovasc Clin, 15(2), 261-75.

Williams, M. y Maresh, C. (2005). Early exercise training in patients older than 65 years compared with that in younger patients after acute myocardial infarction or coronary artery bypass grafting. American Journal of Cardiology, 55, 263-6.

Fecha de recepción del artículo: 15 de mayo del 2007.

Fecha de aceptación del artículo: 10 de junio del 2007.

Fecha de publicación del artículo: 31 de julio del 2007. 